\title{
First Use of Bedaquiline in Democratic Republic of Congo: Two Case Series of Pre Extensively Drug Resistant Tuberculosis
}

\author{
Murhula Innocent Kashongwe1,2, Leopoldine Mbulula², Brian Bakoko ${ }^{3,4}$, Pamphile Lubamba ${ }^{2}$,

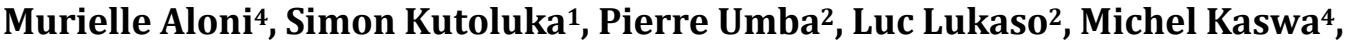 \\ Jean Marie Ntumba Kayembe ${ }^{1}$, Zacharie Munogolo Kashongwe ${ }^{1}$
}

${ }^{1}$ Kinshasa University Hospital, Kinshasa, Democratic Republic of Congo

${ }^{2}$ Action Damien, Centre d'Excellence Damien, Kinshasa, Democratic Republic of Congo

${ }^{3}$ Coordination Provinciale de Lutte contre la Tuberculose, Kinshasa, Democratic Republic of Congo

${ }^{4}$ National Tuberculosis Program, Kinshasa, Democratic Republic of Congo

Email: innocent.kashongwe@unikin.ac.cd

How to cite this paper: Kashongwe, M.I., Mbulula, L., Bakoko, B., Lubamba, P., Aloni, M., Kutoluka, S., Umba, P., Lukaso, L., Kaswa, M., Kayembe, J.M.N. and Kashongwe, Z.M. (2018) First Use of Bedaquiline in Democratic Republic of Congo: Two Case Series of Pre Extensively Drug Resistant Tuberculosis. Journal of Tuberculosis Research, 6, 125-134.

https://doi.org/10.4236/jtr.2018.62012

Received: March 19, 2018

Accepted: June 4, 2018

Published: June 7, 2018

Copyright $\odot 2018$ by authors and Scientific Research Publishing Inc. This work is licensed under the Creative Commons Attribution International License (CC BY 4.0).

http://creativecommons.org/licenses/by/4.0/

\begin{abstract}
In this manuscript the authors have studied the first two patients who were successfully treated with the treatment regimen containing Bedaquiline as second-line drug. The patients were diagnosed with pre-extensively drug-resistant tuberculosis (preXDR TB) whose prognosis was fatal in Democratic Republic of Congo (DRC). Bedaquiline is arguably one of the molecules of the future in the management of ultra-resistant tuberculosis. However, a larger cohort study may help to establish its effectiveness. Case report: $\mathrm{Pa}$ tients 1, 29 years old, with a history of multidrug-resistant TB (MDR-TB) one year previously. He showed signs of TB impregnation again 6 months after the last treatment. Bascilloscopy was positive again. The pre-extensively tuberculosis (pre-XDR TB) diagnosis was made by the Hain test (GenoType MTBDRsl, Hain Lifescience). Patient 2, brother of the first patient, with a history of MDR TB a year before. He had low back pain with right parietal dorso swelling four months after the last treatment. The x-ray of the column showed L4-L5 disc disease. Parietal ultrasound showed a parietal abscess to the right of thoracic vertebrae with fistulization. Surgical biopsy and pus culture confirmed the diagnosis of Pre-XDR Extrapulmonary TB. The treatment regimen was the same for both patients: 6 months with Amikacin (Am) Bedaquiline (Bdq) Prothionamide (Pto) Paraamino Salicylic acid (PAS) Linezolid (Lzd) Cycloserine (Cs) Pyrazinamide (Z) and 14 months with PAS Lzd Cs $Z$. The side effects were minor. Bacteriological controls (smears and cultures) after 20 months of treatment are negative to date.
\end{abstract}




\section{Keywords}

Pre-XDR TB, Pott's Disease, Bedaquiline, Molecular Technique, Loweinstein

Culture

\section{Introduction}

Drug resistance to antituberculosis drugs is a major public health problem; it undermines successes in TB control, especially in developing countries. The treatment of ultra-resistant tuberculosis is not codified. It requires toxic, rare and expensive molecules with poor prognosis. Before the arrival of Bedaquiline in the Democratic Republic of Congo (DRC) in 2015, all patients with extensively drug-resistant TB died by lack of a therapeutic alternative. Community contamination was increased making it difficult to control the infection. This case series describes the first two pre-XDR TB patients treated with bedaquiline-based regimens and their favorable outcomes for the first time in DRC.

\section{Method}

Several patients were diagnosed and died by lack of treatment. Two doses of Bedaquiline (Jansen pharmaceutica) were available through the International Union against Tuberculosis and Respiratory Disease (The Union) in 2015.

Two patients were selected based on their bacteriological profile, extent of radiographic lesions and clinical conditions. The treatment regimen was decided by the Scientific Committee of the National Tuberculosis Program of DRC based on previous history TB treatment and second line drug susceptibility test. A medical assessment including a complete blood count, transaminases, renal function (urea and serum creatinine), lipase, TSH dosage, audiogram and electrocardiogram was mandatory at the beginning of the treatment and then every month. Bedaquiline was administered at a dose of $400 \mathrm{mg}$ for 2 weeks and then $200 \mathrm{mg}$ three times a week for 22 weeks. The doses of the other molecules were adapted according to the weight of the patients (Table 1). A clinical evaluation was done daily and patients were hospitalized for the duration of treatment at the specialized center for the treatment of multidrug-resistant tuberculosis in Kinshasa called "Centre d'Excellence Damien”.

\section{Case Report Series}

Patients 1, 29 years old, who had experienced signs of TB impregnation again six months after a well-managed multidrug-resistant tuberculosis (MDR-TB). $\mathrm{He}$ had received second-line antituberculosis drugs following the short treatment regimen of 4 months made of Kanamycin (Km), Moxifloxacin (Mfx), Prothionamide (Pto), Clofazimine $(\mathrm{Clz})$, Isioniaside $(\mathrm{H})$, Ethambutol (E), Pyrazinamide $(Z)$ and 5 months with $\mathrm{Mfx} \mathrm{Clz} \mathrm{E} \mathrm{Z} \mathrm{with} \mathrm{a} \mathrm{favorable} \mathrm{outcome} \mathrm{(negative}$ bacteriology) after 9 months of treatment. On physical examination it was a 
skinny patient with a right apical cavitary murmur. Chest $\mathrm{x}$-ray showed a right superior lobite excavated and retractable (Figure 1).

Hain test (Genotype MTBDRsl, Hain Lifescience) demonstrated resistance to Fluroquinolones with MUT3C mutation and conserved susceptibility to aminoglycosides. The diagnosis of pre-XDR TB with high resistance to fluroquinolones was made clear. HIV serology was negative. The treatment regimen consisted of two phases: an intensive 6-month phase made of Bedaquiline (Bdq), Prothinamide (Pto), Paraamini Salicylic acid (PAS), Linezolide (Lzd), Cycloserine (Cs) and Pyrazinamide (Z) and 4 months of Amikacin (Am); and a continuation phase of 14 months including only PAA, LZd, Cs, and $\mathrm{Z}$. The treatment regimen was summarized as follows: 4 (Am) 6 (Bdq Pto PAS Lzd Cs Z)/14 (PAS Lzd Cs Z).

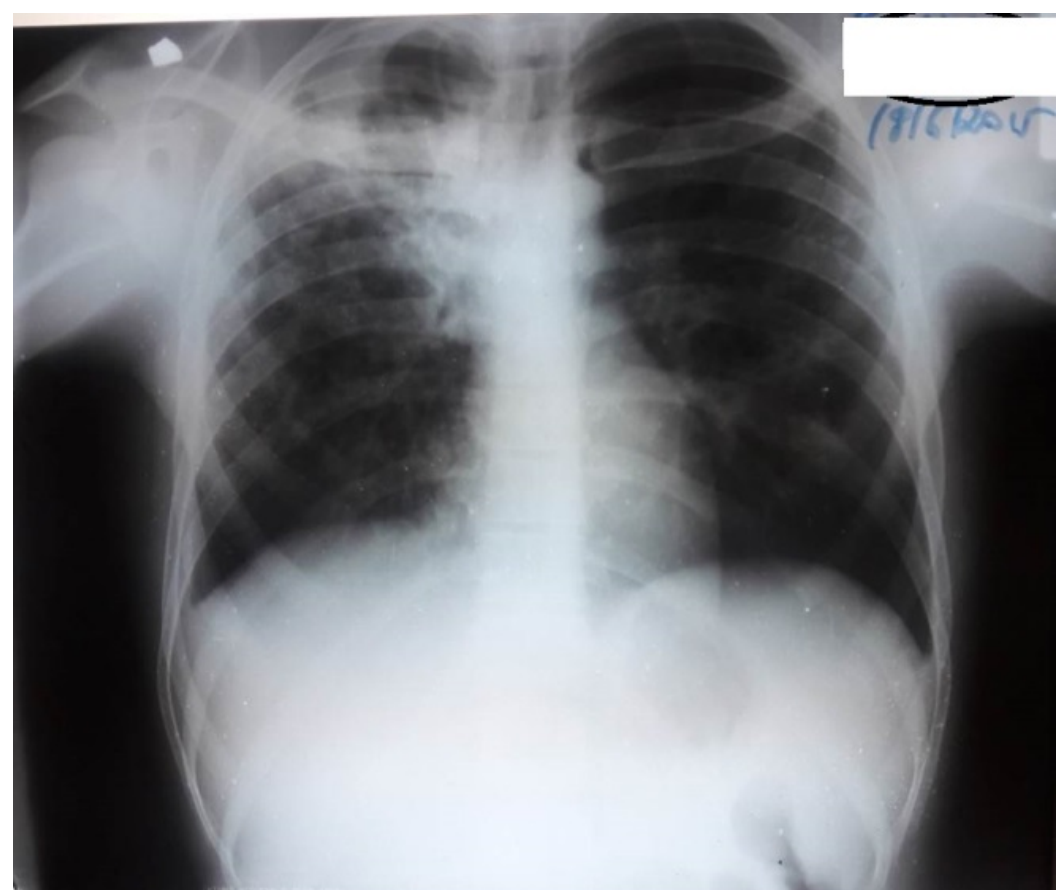

Figure 1. Chest x-ray showing right superior lobar infiltrate excavated and retracted.

Table 1. Protocol used, drugs and dosage.

\begin{tabular}{ccc}
\hline & Protocol used & \\
\hline Drug & Dosage & Type \\
\hline Amikacin (Am) & $15-20 \mathrm{mg} / \mathrm{Kg}$ & $1000 \mathrm{mg}$ ampoule \\
Bedaquiline (Bdq) & $\begin{array}{c}\text { tablets for } 2 \text { weeks, then } 2 \text { tablets three } \\
\text { times a week for } 22 \text { weeks }\end{array}$ & $100 \mathrm{mg}$ tablet \\
Cycloserine (Cs ) & $10-15 \mathrm{mg} / \mathrm{Kg}$ & $250 \mathrm{mg}$ tablet \\
Linesolide (Lzd) & 1 tablet a day & $600 \mathrm{mg}$ tablet \\
PAS (para aminosalicylic acid) & 1 sachet twice a day & 4 Gr sachet \\
Prothionamide (Pto); & $15-20 \mathrm{mg} / \mathrm{Kg}$ & $250 \mathrm{mg}$ tablet \\
Pyrazinamide (Z); & $30-35 \mathrm{mg} / \mathrm{Kg}$ & $400 / 500 \mathrm{mg}$ tablet \\
\hline
\end{tabular}


The adverse effects were: hypersialorrhea, grade 1 diarrhea, rash of the face, and moderate thrombocytopenia isolated at 12 months. There was no abnormality in the ECG during treatment (no QTc prolongation).

Clinical and bacteriological improvements were good with bacteriological negativity at late smear at 6 th month (Table 2). The treatment, started on $11 / 24 / 2015$, ended on $07 / 24 / 2017$ with good evolution (Figure 2).

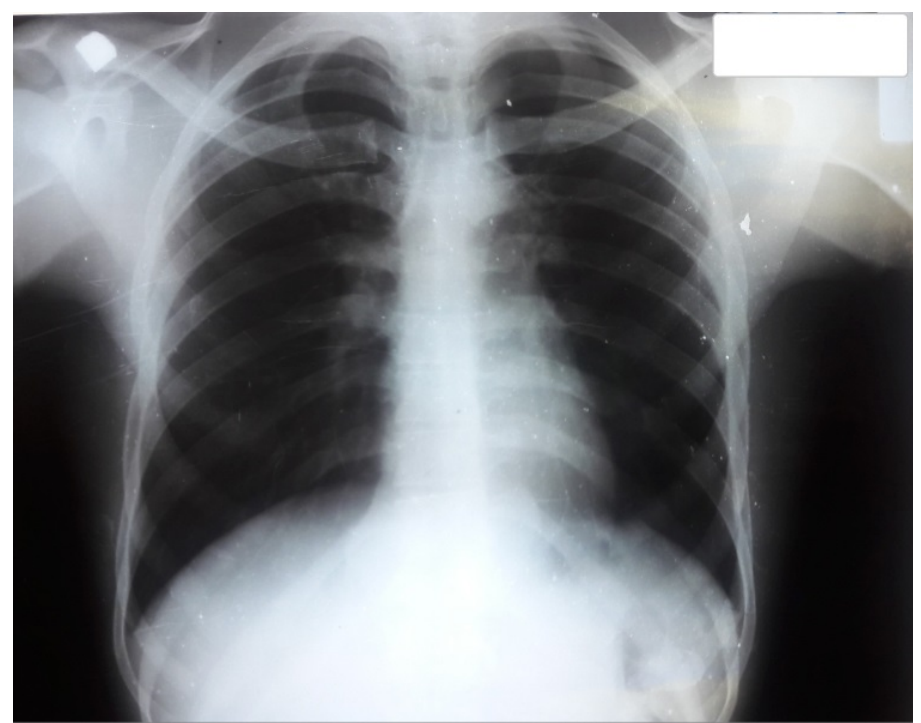

Figure 2. Control X-ray of thorax after twenty month of treatment.

Table 2. Clinical and bacteriological evolution during treatment.

\begin{tabular}{|c|c|c|c|}
\hline \multirow[t]{2}{*}{ Date } & $\begin{array}{l}\text { Sputum Smear } \\
\text { microscopy }\end{array}$ & \multirow[t]{2}{*}{ Culture Patient 1} & \multirow{2}{*}{$\begin{array}{c}\text { Sputum Smear } \\
\text { microscopy and Culture } \\
\text { Patient } 2\end{array}$} \\
\hline & Patient 1 & & \\
\hline Month 0 24/11/2015 & ++ & positive & Negative \\
\hline Month 1 & + & Negative & Negative \\
\hline Month 2 & + & Negative & Negative \\
\hline Month 3 & Negative & - & Negative \\
\hline Month 4 & Negative & Negative & Negative \\
\hline Month 5 & $8 \mathrm{AFB} / 100$ & Negative & Negative \\
\hline Month 6 & $9 \mathrm{AFB} / 100$ & Negative & Negative \\
\hline Month 7 & Negative & contaminated & Negative \\
\hline Month 9 & Negative & Negative & Negative \\
\hline Month 10 & Negative & Negative & Negative \\
\hline Month 12 & Negative & Negative & Negative \\
\hline Month 14 & Negative & Negative & Negative \\
\hline Month 16 & Negative & Negative & Negative \\
\hline Month 18 & Negative & Negative & Negative \\
\hline Month 20 & Negative & Negative & Negative \\
\hline
\end{tabular}


Patient 2, 25 years old, is the brother of the first patient. He had two previous episodes of TB, including one MDR TB in the previous year. He was treated with the second-line treatment regimen called short treatment regimen similar to the one received by patient 1; he was declared cured after 9 months of treatment. He complained about low back pain, recurrent fever, and right parietal dorso-renal swelling four months after the end of TB MR therapy.

Physical examination found a patient with a general condition preserved. IMC was at $22 \mathrm{~kg} / \mathrm{m}^{2}$.

A dorso-parietal right dorso and dorsolumbar kyphosis were evidenced, suggesting the diagnosis of relapse of extrapulmonary TB. Pott's disease in a patient with a recent history of MDR TB.

Radiography of the lumbar spine demonstrated the destruction of the vertebral bodies between L4 and L5 with compression of intervertebral discs (Figure 3).

$\mathrm{X}$-ray of the chest was normal. Parietal ultrasound showed a collection evoking a parietal abscess to the right of thoracic vertebrae with fistulization. Surgical drainage of the abscess was done bringing back two liters and one hundred Milliliters of caseous pus (Figure 4).

Bascilloscopy on pus was negative; medium culture of Loweinstein Jensen identified Mycobacterium tuberculosis with resistance to Fluoroquinolones and Ethambutol. The diagnosis of TB Pre XDR Pott's disease was confirmed. HIV serology was negative. He was treated with similar therapeutic regimen like the first patient. The evolution was marked by the disappearance of symptoms and parietal postoperative cicatrization wound biopsy after 20 months of treatment (Figure 5). Adverse effects were minor with rash of the face; there was no QTc elongation. Post-treatment X-ray revealed bone consolidation. The treatment was started on November 24, 2015 and ended on July 24, 2017.

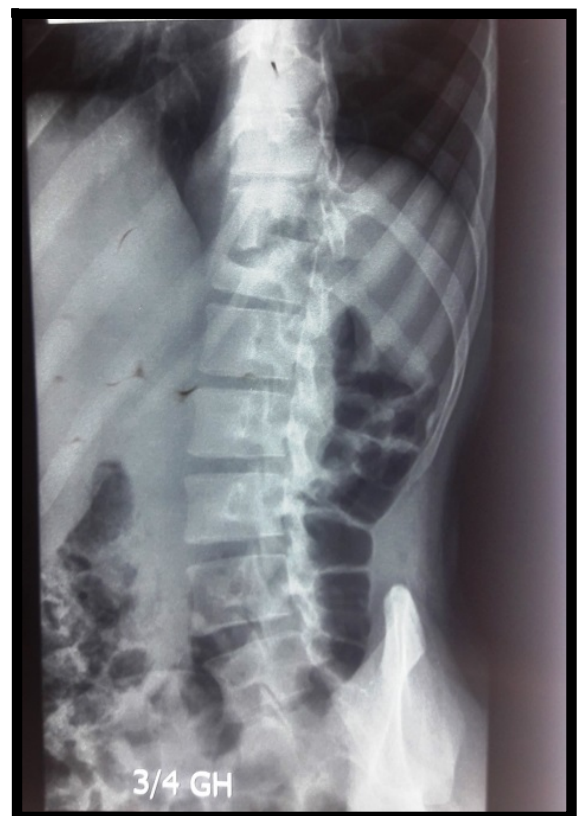

Figure 3. X-ray of the spine showing destruction of L4-L5 vertebral bodies. 


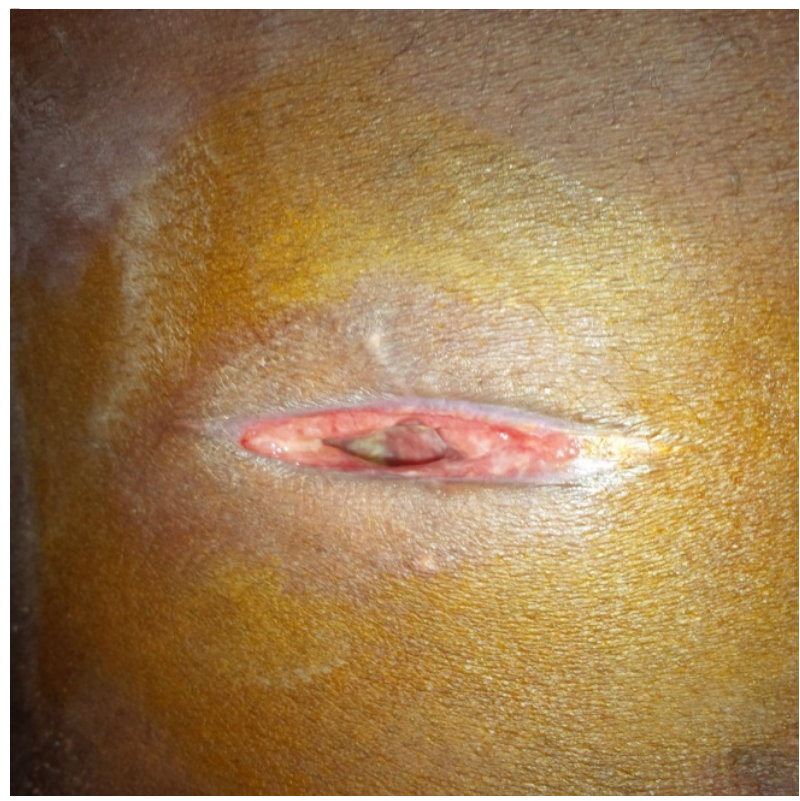

Figure 4. Showing the wound of the surgical incision after drainage of the periosteal abscess right parietal dorso leaving the source of the caseum.

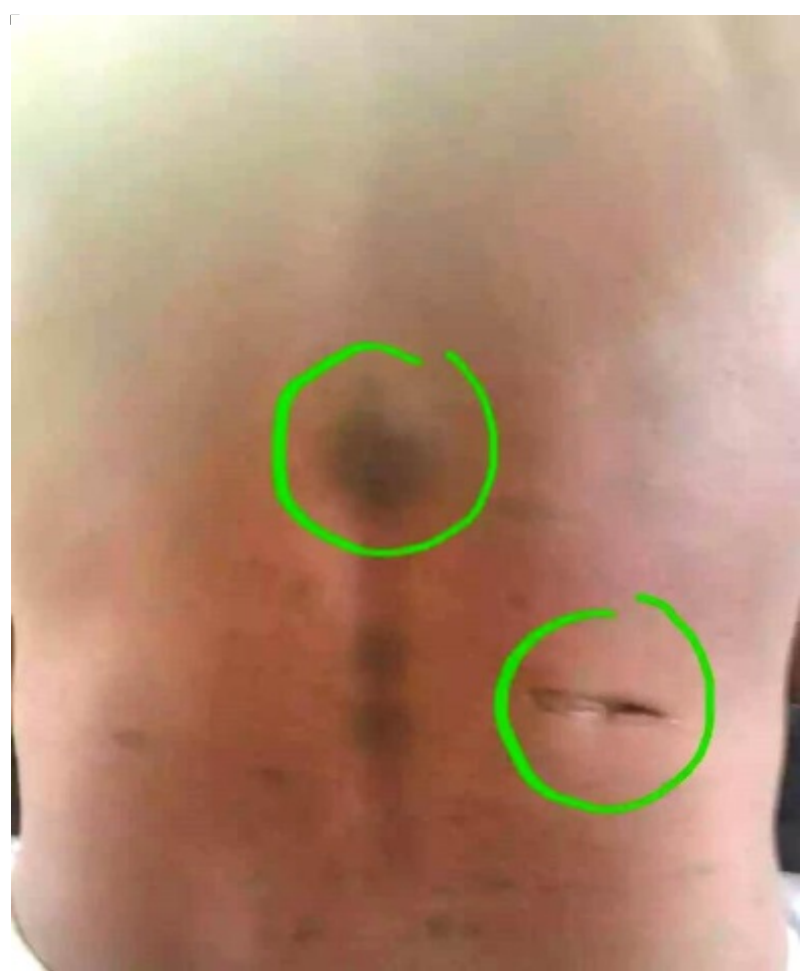

Figure 5. Showing clinical scarring of the spine and parietal abscess.

Both patients have an appointment every month for clinical and bacteriological control during the next two years; Bacteriological control is negative at the 9th month of control post treatment. 


\section{Comment}

In 2015, World Health Organization reported 7579 cases of UR TB from 74 countries (1). Thirty-six percent of reported MR/RR TB cases were pre-XDR or XDR TB patients resulting in unavoidable treatment failure or patient deaths [1]. This reveals the scale of the problem and the importance of early diagnosis for appropriate care. The Democratic Republic of Congo (DRC) is among the 17 African countries that have already reported this form of TB [1] [2] [3].

Pre-extensive drug-resistant tuberculosis (pre-XDR TB) is multidrug-resistant tuberculosis (i.e. tuberculosis with Mycobacterium tuberculosis resistant to rifampicin and isoniazid) plus either fluoroquinolone resistance to at least one of three second-line injectable drugs (amikacin, capreomycin and kanamycin). It is an intermediate stage in antibiotic sensitivity between multidrug-resistant TB and $\mathrm{XDR}-\mathrm{TB}$; this is defined as multidrug-resistant tuberculosis which resists to a fluoroquinolone and at least one of three second-line injectable drugs (amikacin, capreomycin and kanamycin).

$\mathrm{XDR}$ TB is much more serious than MDR TB. Its poor prognosis is mainly due to resistance to fluoroquinolones, a major molecule of second-line treatment [4].

Before the introduction of the molecular tests, the diagnosis in the DRC was made solely on the basis of the culture on solid or liquid medium with realization of the tests of sensitivity. The results were obtained late aggravating the prognosis of the patients; currently, there are various rapid molecular tests including Line Probe Assay (LPA or Hain test) that can give results much faster [5] [6].

In our case series, the diagnosis was made by two different means: the conventional method (Loweinstein Jensen medium culture) and the molecular method (Line probe assay or Hain test). The patient whose diagnosis was made thanks to the culture on solid medium had to wait 4 months before having the confirmation of the diagnosis; the specificity in this case is the extrapulmonary bacteriological confirmation (Pott's disease) which is not that much described in the literature [7] [8] [9] [10]. The history of previous treatments, the notion of close contagion and the almost similar profile of second-line anti-TB sensitivities between the two patients motivated the selection of these two patients for the same third-line treatment regimen.

The therapeutic regimen was built taking into account the recommendations issued by WHO [11] [12] [13]. This treatment regimen was to contain at least 4 new active drugs: Two molecules with bactericidal activities during the intensive phase; two molecules with sterilizing effect to be maintained during the continuation phase and accompanying molecules to protect the action of essential drugs [12].

Thus, depending on the available molecules, the new essential bactericidal molecules were Bedaquiline and Linezolid associated with Amikacin; this latter 
was maintained to preserve the sensitivity. The molecules with sterilizing effect chosen were Cycloserine reinforced by two molecules of the previous treatment regimen by lack of alternative, which are Prothionamide and pyrazinamide. They were reinforced by Para Aminosalycilic Acid (PAS) as an accompanying molecule. It was a wise choice given the urgency and taking into account the available molecules. In fact, the DRC recorded a large number of deaths in the pre XDR diagnosed by lack of alternative treatment [2].

Nevertheless, this treatment regimen was risky. Patients already had resistance to rifampicin. Sensitivity to Isoniazid (INH) was not known. In case of high resistance to INH, there is cross-resistance with thionamides [12]; the lack of information regarding the sensitivity to prothionamide already used in the short treatment regimen of second-line for MDR-TB [13] motivated its reinforcement by Cycloserine and PAS. The injectable used previously was Kanamycin; Amikacin was chosen considering its sensitivity preserved to injectables. Evolution would not have been the one observed if there were cross-resistance.

Several major adverse effects are described in the literature [14]. In our case series, there have been mainly minor digestive disorders that have been relieved by antiemetics and anti-diarrhea drugs; these digestive effects were attributed to the combination PAS, Prothionamide and Pyrazinamide. The haematological disorder observed, an isolated trombocytopenia, was due to linezolid, which was amended without treatment.

The relatively good compliance to treatment is probably related to the good condition at first, to hypercaloric nutrition with weight gain under treatment, and early management in a specialized center of adverse effects observed.

It is recommended to assess a complete the treatment susceptibility at the starting of TB treatment; this assessment is crucial to ensure the effective treatment for patients. We can improve the treatment of TB by performing drug susceptibility test (DST) in all TB patients.

\section{Conclusion}

Bedaquiline is without doubt one of the molecules of the future in the management of extensively drug-resistant tuberculosis. However, a larger cohort study may help to establish its effectiveness. Tuberculosis always succeeds in developing mechanisms for antibiotic resistance. The use of new molecules must be done in appropriate treatment regimens that take into account sensitivity tests of all second-line molecules; this could enable their protection and cure of all forms of tuberculosis.

\section{Acknowledgements}

The authors grateful thank to Action Damien Foundation for the financial management of hospitalized patients and gratefully thank to "The Union" who give them de first Bedaquiline. Gratefully thank also to Dr alberto Piubello for the training of doctors and nurses in CEDA. 


\section{Funding}

This work received no financial assistance from any funding agency in the public, commercial, or non-profit sectors.

\section{Competing Interest}

The authors declare that they do not have any financial interest with the information contained in this paper.

\section{Ethics Approval}

The study protocol was approved by the national committee of NTP of DRC.

\section{References}

[1] World Health Organization (2016) Global Tuberculosis Report 2016. WHO, Geneva. http://www.who.int/tb/publications/global_report/en/

[2] National Tuberculosis Program of DR Congo (2016) Rapport du groupe thématique sur la tuberculose pharmacorésistante pour la revue externe à mi-parcours, PNLT RD Congo.

[3] Kashongwe, Z.M., Muyembe, T.J.J., Wembanyama, H., Kalonga, R. and Ngoyi, T.N. (1998) Tuberculose Multirésistante à Kinshasa: étude rétrospective de 24 cas chronique. Congo Médical Aout, 6, 314-317.

[4] Kim, D.H., Kim, H.J., Park, S.K., et al. (2011) Treatment Outcome ans Survival Based on Drug Resistant Patterns in Multidrug Resistant Tuberculosis. American Journal of Respiratory and Critical Care Medicine, 183, 278-279.

[5] Theron, G., Peter, J., Richardson, M., Barnard, M., Donegan, S., Warren, R., Steingart, K.R. and Dheda, K. (2014) The Diagnostic Accuracy of the GenoType MTBDRsl Assay for the Detection of Resistance to Second-Line Anti-Tuberculosis Drugs. Cochrane Database of Systematic Reviews, No. 10, Article ID: No. CD010705.

[6] Clouse, K., et al. (2012) Implementation of Xpert MTB/RIF for Routine Point-of-Care Diagnosis of Tuberculosis at the Primary Care Level. South African Medical Journal, 102, 805-807. https://doi.org/10.7196/SAMJ.5851

[7] Vyazovaya, A., Mokrousov, I., Solovieva, N., Mushkin, A., Manicheva, O., Vishnevsky, B., Zhuravlev, V. and Narvskaya, O. (2015) Tuberculous Spondylitis in Russia and Prominent Role of Multidrug-Resistant Clone Mycobacterium tuberculosis Beijing B0/W148. Antimicrob Agents Chemother, 59, 2349-2357. https://doi.org/10.1128/AAC.04221-14

[8] Ko, Y., Lee, H.Y., Lee, Y.S., et al. (2014) Multidrug-Resistant Tuberculosis Presenting as Miliary Tuberculosis without Immune Suppression: A Case Diagnosed Rapidly with the Genotypic Line Probe Assay Method. Tuberculosis and Respiratory Diseases, 76, 245-248. https://doi.org/10.4046/trd.2014.76.5.245

[9] Park, C.S., et al. (2014) Bronchoesophageal Fistula with Gastric Perforation. World Journal of Gastrointestinal Surgery, 6, 253-258.

http://www.wjgnet.com/esps/helpdesk.aspx https://doi.org/10.4240/wjgs.v6.i12.253

[10] Das, S.K., Das, A., Gangopadhyay, A. and Sinha, A.K. (2012) Primary Disseminated Extrapulmonary Multidrug Resistant Tuberculosis. Indian Journal of Medical Mi- 
crobiology, 30, 364-366. https://doi.org/10.4103/0255-0857.99509

[11] WHO (2016) Treatment Guidelines for Drug-Resistant Tuberculosis. Geneva, World Health Organization.

http://www.who.int/tb/areas-of-work/drug-resistant-tb/treatment/resources/en/

[12] Scardigli, A., Caminero, J.A., Sotgiu, G., Centis, R., D’Ambrosio, L. and Migliori, G.B. (2016) Efficacy and Tolerability of Ethionamide versus Prothionamide: A Systematic Review. The European Respiratory Journal, 6, 946-952.

[13] WHO (2016) Shorter MDR TB Regimen: Frequently Asked Questions about the Implementation of the New WHO Recommendation on the Use of the Shorter MDR-TB Regimen under Programmatic Conditions. World Health Organization, Geneva.

http://www.who.int/tb/areas-of-work/drug-resistant-tb/treatment/FAQshorter_MD R_regimen.pdf

[14] WHO (2016) Active TB Drug-Safety Monitoring and Management (aDSM). WHO. http://www.who.int/tb/areas-of-work/drug-resistant-tb/treatment/pharmacovigilan ce/en/ 